\title{
Avaliação da proposta de um modelo de sistema de ajuda online para a UFMG Virtual
}

\author{
Letícia Capelão' ${ }^{1}$ Raquel Prates², Leonardo Freitas ${ }^{3}$, Heider Maciel ${ }^{3}$ \\ Faculdade de Letras ${ }^{1}$, Laboratório de Ciência da Computação ${ }^{2}$, Departamento de \\ Ciência da Computação ${ }^{3}$. \\ Universidade Federal de Minas Gerais (UFMG) - Belo Horizonte, MG - Brazil \\ \{leticiac, rprates\}@dcc.ufmg.br, \{leonardof, hrmaciel\}@lcc.ufmg.br
}

\begin{abstract}
Learning environments online, especially Moodle, are computational systems that offer a wide variety of resources and tools to support teaching. They are used for sharing information and resources, as well as to promote socialization, integration and construction of knowledge by their users. Therefore, online help systems are essential in these envirnoments in order to provide a better communicability of the designers' intentions and decisions. This paper proposes a model for an online help system to UFMG Virtual (Moodle Platform of UFMG), based on Semiotic Engineering theory.
\end{abstract}

Resumo. Os ambientes de aprendizagem on-line, especialmente o Moodle, são sistemas computacionais que oferecem uma grande variedade de recursos $e$ ferramentas para apoio ao ensino, tanto para fins de compartilhamento de informações, quanto para promoverem a socialização, integração $e$ construção do conhecimento pelos seus usuários. Diante disso, os sistemas de ajuda online são essenciais para seu uso como um meio de possibilitar uma melhor comunicabilidade das intenções do designer. Este artigo propõe um modelo de sistema de ajuda para UFMG Virtual (plataforma Moodle do Portal UFMG), fundamentando na teoria da Engenharia Semiótica.

\section{Introdução}

A UFMG Virtual é a plataforma de apoio ao ensino presencial, semipresencial e a distância da Universidade Federal de Minas Gerais (UFMG) que utiliza o ambiente de aprendizagem on-line Moodle e foi escolhida como ambiente de ensino para proposta deste sistema de ajuda.

Os ambientes de aprendizagem on-line possibilitam o uso e integração de diferentes mídias, linguagens e recursos para armazenamento, comunicação e colaboração, que visam estimular a interação entre pessoas e grupos. O Moodle tem sua concepção pautada na construção compartilhada do conhecimento, onde seus participantes (alunos, professores, tutores etc) aprendem através da socialização e colaboração. Devido à complexidade inerente aos processos de ensino e aprendizado e aos diversos recursos e funcionalidades oferecidos pelos ambientes de apoio ao ensino, especialmente o Moodle, é emergente a necessidade de apoiar o seu uso. Contudo, pesquisas já identificaram lacunas no sistema de ajuda oferecido pela UFMG Virtual e o Moodle, assim como os sistemas de ajuda tradicionais (tutoriais, manuais, FAQs etc), 
em função da qualidade do próprio recurso oferecido ou em virtude do próprio usuário (que não faz uso do sistema de ajuda). Com isso, o objetivo deste trabalho é propor um novo modelo de sistema de ajuda a partir de uma metolodogia fundamentada na teoria da Engenharia Semiótica (EngSem) para Interação Humano-Computador.

\section{Justificativa}

\subsection{UFMG Virtual}

A UFMG Virtual é um projeto desenvolvido pelo Laboratório de Computação Científica (LCC) da UFMG que envolve a instalação e customização do Moodle e sua integração ao Portal minhaUFMG. Este projeto se iniciou em março de 2007. Hoje este projeto utiliza a versão 2.0 do Moodle, integrada ao Sistema Acadêmico da UFMG e ao Diário de Classe Eletrônico.

O Moodle oferece uma gama de possibilidades de configuração de cursos e recursos que apoiam a EaD. As estatísticas divulgadas mostram que o Moodle está instalado em 84.474 sites e tem 71.788 .175 usuários registrados no mundo. O Brasil ocupa terceira posição de número de sites Moodle registrados, com 6.286. Todavia, apesar da flexibilidade e grande difusão de uso, através de pesquisa realizada por Capelão et al (2011) foram identificados problemas na forma como a comunicação da lógica proposta por seus criadores é recebida por seus usuários, o que na EngSem se caracterizam como rupturas de comunicação. Alguns usuários buscaram por algum recurso de ajuda no sistema a fim de transpor os problemas de comunicação encontrados. Entretanto, não encontraram a ajuda necessária e a interação com o sistema foi comprometida. O sistema de ajuda oferecido foi o do próprio Moodle, com poucas informações de ajuda para os professores, que têm que investir muito tempo no aprendizado para uso apropriado de seus recursos ou o fazem a partir de recursos triviais. O sistema fica então subutilizado e seus recursos para apoiar e motivar os processos de ensino-aprendizagem não são explorados.

\subsection{Metodologia}

Um sistema que apresenta boa comunicabilidade, deve ser capaz de transmitir a intenção do designer através de sua interface [de Souza, 2005]. Entretanto, diante dos diversos serviços disponibilizados por estes ambientes de aprendizagem e das várias possibilidades de uso pedagógico, somente a interação dos usuários com o sistema não é suficiente para que eles façam uso dos recursos de forma apropriada. Dentre os recursos de ajuda mais comuns e utilizados, destacam-se: manuais do usuário, tutoriais, treinamentos, helpdesk e sistemas de ajuda on-line. Os diversos tipos de ajuda foram apresentados, descritos e discutidos por Silveira e Leite (2009): ajuda embutida, assistência contextual, descrição da aplicação, diálogo, dicas em elementos de interação, notas, perguntas frequente (FAQ), tabela de conteúdo e índices e tutoriais on-line.

Conforme identificado por Silveira et al. (2003), os sistemas de ajuda online "acabam por não ajudar muito os usuários" e apresentam algumas razões deste problema da ajuda que envolvem os projetistas dos sistemas: "a falta de tempo ou de planejamento para desenvolvimento de um sistema de ajuda, a excessiva confiança ou expectativa em relação a intuitividade da interface construída; ou ainda, uma ingênua aceitação dos padrões correntes." Diante disso, criou-se uma cultura de desuso ou quase 
nenhum uso dos sistemas de ajuda pelos usuários. Kearsley (1998) citado por Silveira et al. (2000) já apresentava alguns motivos para esse "descaso": falta de informação específica, indisponibilidade de informação necessária, imprecisão das informações encontradas e dificuldade em trocar de contexto entre a aplicação e o sistema de ajuda. A partir destes estudos, Silveira (2002) e Silveira et al. (2003) propuseram então um novo método para construção de sistemas de ajuda.

O Colabora UFMG Virtual está sendo desenvolvido a partir deste método proposto pelas pesquisadoras que se baseia em dois pilares: a comunicabilidade e a técnica retórica de layering da abordagem minimalista. $O$ método propõe que a interação do usuário com o conteúdo do sistema de ajuda seja feita a partir das expressões de comunicabilidade (O que é?, Para quê serve?, Como funciona? etc). Esta técnica de layering (ajuda em camadas) [Farkas, 1998] visa apresentar o conteúdo do sistema de ajuda em camadas interligadas com pequenas porções de conteúdo que são consultadas pelos usuários, dependendo de sua escolha e necessidade, até receber as informações desejadas. Pela abordagem minimalista, o conteúdo é descrito de forma resumida e objetiva, apresentando ao usuário somente a informação necessária para aquele contexto de ajuda. Este método se configura como uma proposta moderna de sistema de ajuda que busca se enquadrar nas reais necessidades de interação e informação do usuário.

\section{Trabalhos Relacionados}

Diversos estudos e pesquisas já foram desenvolvidos e publicados por pesquisadores para construção de sistemas de ajuda fundamentados na EngSem: modelo e arquitetura de help online [Silveira et al., 2000]; método para construção de sistema de ajuda online utilizando a técnica de layering em uma abordagem minimalista [Silveira, 2002]; estudo de caso para aplicação do método proposto por Silveira (2002) contendo os passos para a sua construção com exemplos de cada etapa deo método [Silveira et al., 2003]; aplicação de uma avaliação informal, de caráter exploratório, do esboço de um sistema de ajuda proposto pela EngSem, onde as expressões de comunicabilidade foram confirmadas e novas expressões foram identificadas [Salgado e de Souza, 2004]; abordagens para aperfeiçoar os sistemas de ajuda [Farkas, 1998; Silveira, 2002]; conceitos importantes da MoLIC e os princípios de design relacionados [Silva, 2005]; guia prático que descreve como definir a interação do usuário com o sistema de acordo com a 2a edição da MoLIC [Silva e Barbosa, 2007]; análise de sistemas de ajuda disponibilizados em ambientes de aprendizagem colaborativa [Silveira e Leite, 2009]; forma de organização das informações em sistemas de ajuda on-line a partir do contexto do usuário, o diálogo do usuário-sistema e apresentação de pequenas porções de informação a cada solicitação de ajuda [Santos Jr, 2009]; ferramenta que permite a organização das informações de ajuda (de acordo com as expressões e camadas) e, a partir destas informações gera os modelos de IHC resumidos [Silva e Farias, 2010]; ferramenta para auxiliar na construção de conteúdo de sistemas de ajuda e na geração de versões simplificadas de modelos de Interação Humano-Computador (IHC) [Goebel e Chichelero, 2010]; proposta de uma arquitetura e um protótipo de ferramenta para a implantação de um sistema de ajuda no formato peer help (ajuda em pares) [Leite e Silveira, 2011]. 


\section{Metodologia}

A proposta do desenvolvimento do sistema de ajuda para o Colabora UFMG Virtual foi organizada a partir da identificação das principais rupturas de comunicação na visão de professores [Capelão et al., 2011]; pesquisa de trabalhos relacionados sobre sistema de ajuda e proposta de novas expressões de comunicabilidade a partir da consulta ao Helpdesk minhaUFMG ${ }^{1}$.

O método proposto por Silveira (2002) e Silveira et al. (2003) descreve os passos na construção de um sistema de ajuda online: (1) construção dos modelos de design de IHC considerando-se questões específicas relacionadas à ajuda; (2) geração de um rascunho do conteúdo da ajuda; (3) refinamento do conteúdo e especificação dos pontos de recorrência; (4) construção do módulo de ajuda geral; (5) conexão dos pontos de acesso à ajuda aos elementos de interface; (6) testes preliminares; (7) análise de uso durante a avaliação de comunicabilidade e (8) refinamento ou redesign do conteúdo da ajuda. O método é ilustrado na Figura 1.

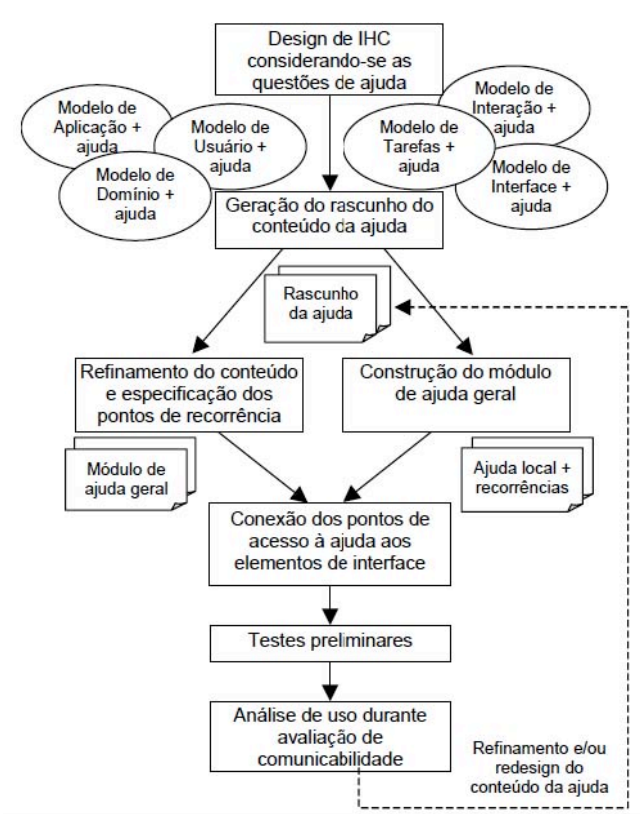

Figura 1. Método de construção de sistema de ajuda on-line conforme proposto por Silveira, De Souza e Barbosa (2003).

O Colabora UFMG Virtual seguiu também a proposta de organização da informação em sistemas de ajuda apresentada por Santos Jr. (2009): acesso, disposição e relevância das informações. Esta organização está detalhada na seção seguinte (Implementação e Resultados). As informações do conteúdo de ajuda foram construídas a partir dos modelos de IHC propostos em Silveira (2002), de forma a contemplar as informações mais relevantes para o usuário.

\footnotetext{
${ }^{1}$ O Helpdesk minhaUFMG tem como objetivo oferecer suporte a toda comunidade da UFMG (alunos, professores e funcionários) nas várias aplicações do Portal minhaUFMG, dentre elas: UFMG Virtual (Moodle), cadastro de usuários, correio eletrônico, agenda.
} 


\section{Implementação e Resultados}

A implementação do sistema de ajuda contemplou as etapas propostas por Silveira et al. (2003), conforme Figura 1. Os oito passos que compõem a metodologia proposta estão descritos a seguir.

Para fins de escopo de construção e avaliação do sistema de ajuda, optou-se pela funcionalidade Tarefa do tipo Modalidade Avançada de Carregamento de Arquivos (MACA), na visão de uso de professores. Este tipo de tarefa é utilizado pelos professores e alunos para envio de atividades pela UFMG Virtual, permitindo o envio de um ou mais arquivos, com possibilidade de revisão e registro de anotações pelos alunos para cada tarefa submetida. Esta tarefa possui uma tela de configuração de valores para configuração geral (nome, data de entrega, envio com atraso), configuração de notas (escalas), configuração para o tipo MACA (tamanho de arquivos, registro de anotações etc) e configurações comuns de módulos (grupos e agrupamentos).

\subsection{Design de IHC}

Esta etapa de design de IHC para o Colabora UFMG Virtual foi elaborada considerando-se questões específicas relacionadas à ajuda do modelo de tarefa e modelo de interação, conforme método proposto por Silveira et al. (2003). A seguir apresentamos a descrição de cada um dos modelos. Os diagramas não foram incluídos neste artigo por razões de limitação de espaço.

O modelo de tarefas apresenta informações relativas às tarefas executadas pelos usuários na aplicação. O modelo de tarefas utilizado na EngSem representa a estrutura hierárquica das tarefas, assim como as estruturas de sequência e iteração, além de tarefas alternativas, independentes de ordem, opcionais e ubíquas. [Barbosa e Silva, 2010; Silveira e de Souza, 2002]. A partir do escopo do Moodle selecionado para este trabalho foi elaborado um modelo de tarefas hierárquico.

"O modelo de interação contém informações sobre as formas de interação possíveis na aplicação, ou seja, sobre como efetivamente se dá a conversa para realizar uma determinada tarefa na aplicação." [Silveira, 2002]. O modelo de interação deste trabalho foi desenvolvido a partir da linguagem MoLIC (Modeling Language for Interaction as Conversation). A MoLIC é uma linguagem para a modelagem da interação seguindo a metáfora da interação como uma conversa proposta por Barbosa e Paula (2003). Ela é composta por três artefatos: (1) o diagrama de metas (objetivos), (2) o esquema conceitual de signos e (3) o diagrama de interação. O primeiro artefato, o digrama de metas (objetivos), indica o que os usuários devem fazer na aplicação. Para o escopo deste trabalho, temos: Eu (usuário no papel Professor) quero incluir uma atividade do tipo Tarefa como Modalidade Avançada de Carregamento de Arquivos para que os alunos possam enviar um trabalho. O esquema conceitual de signos (segundo artefato) define e organiza os conceitos envolvidos no sistema, especialmente aqueles que são apresentados aos usuários na interface. Inclui as informações relacionadas a cada ação do usuário, sistema ou interlocutor externo que afete a interação usuário-sistema. Este esquema foi elaborado para os signos relacionados à inserção da tarefa do tipo MACA para cada um dos blocos: configuração geral (8 signos), configuração de notas (2 signos), configuração da modalidade avançada de carregamento de arquivos (7 signos) e configurações comuns de módulos (4 signos). Apresenta o conteúdo dos signos, restrições de valores, valores default e mecanismos de 
prevenção e tratamento de rupturas para cada signo. O diagrama de interação (MoLIC) representa como os objetivos poderão ser atingidos durante a interação, sendo uma ponte entre os objetivos dos usuários e o projeto da interface. Ele foi elaborado na ferramenta MolicDesigner e apresenta a interação do usuário professor para a meta: Inserir tarefa do tipo Modalidade Avançada de Carregamento de Arquivos (MACA).

\subsection{Geração do Rascunho do Conteúdo de Ajuda}

A geração o rascunho do conteúdo da ajuda contemplou a elaboração de templates contendo as respostas minimalistas para cada expressão de comunicabilidade; geração de respostas para cada par expressão-elemento (signo, tarefa, cursos alternativos de ação e ações propriamente ditas). [Silveira et al. 2003].

A partir da análise das expressões de comunicabilidade propostas por Silveira (2002), Silveira et al. (2003), Leite e Silveira (2011) e Santos Jr. (2009), novas expressões foram propostas para serem utilizadas e avaliadas no Colabora UFMG Virtual. Essas novas expressões foram propostas também a partir da avaliação de dúvidas mais frequentes apresentadas pelos usuários da UFMG Virtual (Moodle) ao Helpdesk minhaUFMG. As expressões usadas foram: São elas: O que é?, Para que serve?, Como funciona?, Qual a aplicação pedagógica?, Por que devo usar?, O que fazer?, O que não fazer?, Como faço isso?, Por que não funciona?, Socorro! Foram descritas respostas minimalistas para cada uma destas expressões de comunicabilidade, considerando-se a atividade Tarefa e, especificamente, Tarefa do tipo MACA.

A proposta inicial para a geração do rascunho do conteúdo de ajuda seria utilizar a ferramenta proposta por Goebel e Chichelero (2004) que "embora utilize a abordagem da Engenharia Semiótica, visa diminuir o esforço do designer através da utilização de um fluxo inverso, que elimina a necessidade de se preencher os modelos de IHC, focando-se diretamente nas expressões de ajuda". Contudo, não foi utilizada pois não contemplava todas as expressões de comunicabilidade para ajuda e não oferecia a funcionalidade para expandi-las com novos templates de resposta. $\mathrm{O}$ rascunho do conteúdo de ajuda foi então elaborado a partir da ferramenta Microsoft Word.

\subsection{Refinamento do Conteúdo e Especificação das Recorrências}

Foram selecionados os elementos principais sobre os quais os usuários pudessem ter dúvidas, relacionados à atividade Tarefa e Tarefa do tipo MACA. Os rascunhos de respostas gerados foram revisados e refinados de forma a possibilitar uma melhor comunicação para os usuários com foco em respostas minimalistas e direcionadas às expressões de comunicabilidade, assim como identificar os possíveis pontos de recorrência. Estes pontos permitem o aprofundamento das informações de ajuda de um determinado tópico. Com isso, foram gerados os elementos textuais de respostas adicionais às expressões de conteúdo de ajuda associados, caracterizando estes pontos de recorrência. (ver Figura 3)

\subsection{Construção do Módulo de Ajuda Geral}

O módulo de ajuda geral foi elaborado com informações sobre o domínio e a aplicação (UFMG Virtual/Moodle), além de explicações sobre como o sistema de ajuda funciona, os elementos de sua interface e problemas encontrados fora do contexto da aplicação 
(conexão, acesso). Esse conteúdo foi disponibilizado na primeira aba "Colabora UFMG Virtual" que pode ser visualizada na Figura 2 na seção seguinte.

\subsection{Conexão dos Pontos de Acesso à Ajuda aos Elementos de Interface}

Nesta fase, o conteúdo da ajuda geral e local (expressões e respostas correspondentes) foram disponibilizados na interface do sistema de ajuda Colabora UFMG Virtual.

O acesso ao Colabora UFMG Virtual é feito através de um link "Acesse o Colabora UFMG Virtual (Sistema de Ajuda Moodle)" disponível no portal MinhaUFMG. O sistema de ajuda está sendo construído em uma turma da UFMG Virtual (Moodle versão 2.0.10) e utiliza um tema desenvolvido pela equipe do Laboratório de Computação Científica (LCC/UFMG). Foi adicionada também a este tema uma nova opção de exibição do formato do curso "tópico único (abas)" com as seguintes abas: Colabora UFMG Virtual, Mural de Recados, UFMG Virtual, Comunicando e Interagindo, Fóruns e Tarefas. A interface pode ser vista na Figura 2.

Seguimos a proposta de organização da informação em sistemas de ajuda apresentada por Santos Jr. (2009). Ela considera três aspectos principais para a comunicação usuário-sistema: o acesso (como o usuário inicia o processo de ajuda), a disposição (como o usuário visualiza a informação) e a relevância das informações. $O$ acesso ao conteúdo do Colabora UFMG Virtual está sendo feito através das expressões de comunicabilidade disponibilizadas em cada uma das abas (ver Figura 2). O conteúdo do sistema de ajuda (respostas às expressões a partir do acesso pelos links de cada uma das expressões de comunicabilidade) é apresentado em janelas individuais (páginas HTML) onde os novos conteúdos podem ainda ser aprofundados através de pontos de recorrência (hiperlinks). Estes pontos de recorrência estão exemplificados na Figura 3.

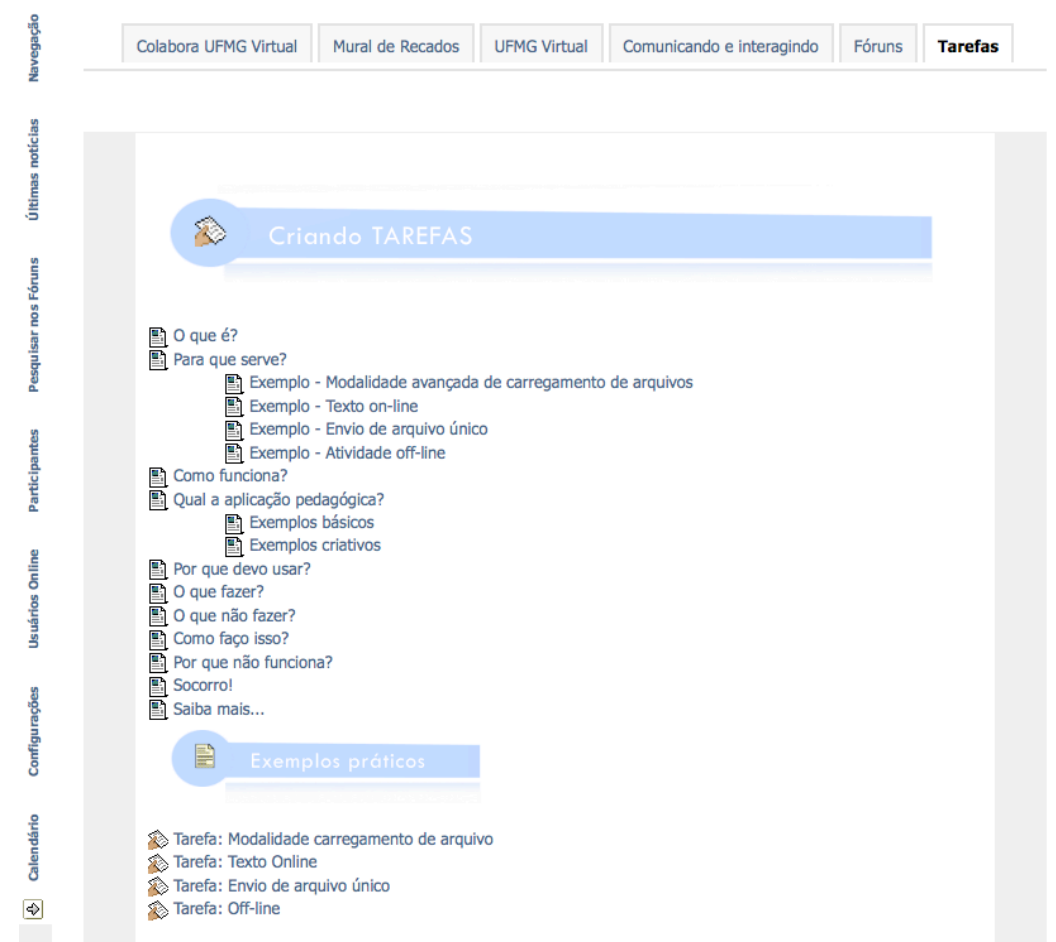

Figura 2. Tela da interface do sistema de ajuda on-line Colabora UFMG Virtual. 


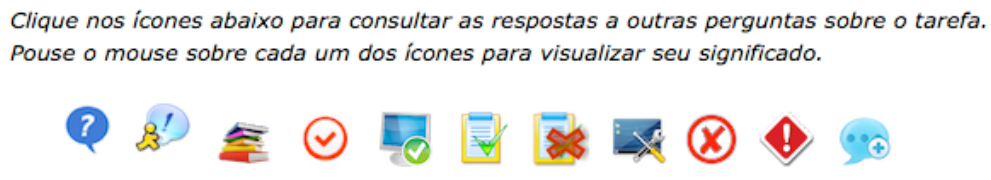

Figura 3. Exemplos de pontos de recorrência (links internos)

\subsection{Testes preliminares}

Foram realizadas avaliações preliminares a fim de verificar a consistência do conteúdo da ajuda geral e local, assim como as conexões das expressões, suas respostas e pontos de recorrência. Estas avaliações foram realizadas pela própria equipe responsável pela construção do sistema de ajuda. Os problemas encontrados (inconsistências em pontos de recorrência, conteúdo ambíguo) foram corrigidos e as alterações necessárias foram realizadas.

\subsection{Análise de Uso durante Avaliação de Comunicabilidade}

Esta etapa de análise de uso é realizada durante a avaliação de comunicabilidade. Esta etapa não foi realizada. Para isto, aplicaremos 0 método de avaliação de comunicabilidade (MAC) na interface sem o sistema o sistema de ajuda e com o sistema de ajuda. Os resultados serão comparados e analisados. O MAC é aplicado em ambiente controlado com usuários e visa avaliar se a interface identificando os possíveis problemas de comunicabilidade a partir da recepção do conteúdo da ajuda (metamensagem do designer) pelo usuário [de Souza, 2005].

\subsection{Refinamento e/ou Redesign do Conteúdo da Ajuda}

O redesign do conteúdo de ajuda será realizado a partir dos resultados obtidos no MAC. Com isso, serão avaliadas as alterações necessárias na interface, no conteúdo e conexões da interface. Após estes ajustes ainda são necessários novos testes com os usuários até que problemas significativos não sejam mais encontrados.

\section{Conclusões}

No contexto de IHC, a avaliação da proposta deste sistema de ajuda no escopo definido pretende contribuir para uma futura e potencial melhor comunicabilidade da interface da UFMG Virtual; um melhor entendimento da solução proposta pelo projetista na plataforma Moodle da UFMG Virtual; melhoria das qualidades de uso de acessibilidade, usabilidade e comunicabilidade do sistema Moodle. No contexto educacional, a implementação de um sistema de ajuda on-line a partir desta metodologia pode contribuir para um maior uso do plataforma Moodle no âmbito educacional e por seus usuários, em seus diferentes papéis (professores, tutores, alunos etc). Este trabalho também possibilita a aplicação e avaliação do método proposto por [Silveira, 2002] em um sistema real, num contexto educacional, para um ambiente de aprendizagem on-line. 
Este trabalho apresentou facilidades e dificuldades durante o seu desenvolvimento. Dentre as facilidades destacamos o apoio dos pesquisadores de outras instituições de ensino que também estão envolvidos na temática de sistemas de ajuda online. Além disso, há diversas publicações e trabalhos desenvolvidos que apoiaram a fundamentação teórica e a aplicação do método. Dentre as dificuldades, podemos destacar: a construção de um sistema de ajuda online e desenvolvimento de suas etapas (elaboração de modelos, geração do rascunho de ajuda etc) para uma aplicação complexa já projetada por terceiros, concluída e em uso; criação dos arquivos HTML e seus links internos feitos de forma manual; a ferramenta para elaboração do conteúdo de ajuda de Santos Jr. (2009) contempla apenas expressões validadas pela pesquisa e não oferece links internos para o conteúdo de ajuda contido nas expressões "o que é" e "para que serve". Em uma implementação de maior porte, a utilização de uma ferramenta específica e integrada à UFMG Virtual é essencial com intuito de tornar o trabalho mais eficiente, minimizar a ocorrência de erros, assim como manter o conteúdo arquivado e organizado.

Pretende-se desenvolver o sistema de ajuda on-line para todos os signos da interface da tela da tarefa do tipo MACA e realizar testes de avaliação de comunicabilidade (MAC) com professores. Os testes serão realizados na interface contendo o sistema de ajuda já presente na UFMG Virtual e, posteriormente, utilizando a interface contendo a implementação do sistema de ajuda proposto neste trabalho. A partir disso, será possível fazer uma comparação e consequente validação do sistema de ajuda. Pretende-se também organizar o conteúdo do sistema de ajuda utilizando o recurso "Livro" a fim de avaliar os quesitos de usabilidade. Contudo, essa proposta deve ser avaliada com os usuários, assim como os seus resultados para não comprometer a comunicabilidade

Agradecimentos: Agradecemos à FUNDEP, pelo apoio financeiro concedido para a realização desta pesquisa; ao diretor do LCC/UFMG, professor Márcio Luiz Bunte de Carvalho e à coordenadora do GIZ/UFMG, professora Juliane Côrrea, pelo apoio ao desenvolvimento do projeto; aos pesquisadores Milene Selbach Silveira, Letícia Lopes Leite, Simone Diniz Junqueira Barbosa e Bruno Santana da Silva por toda a atenção, motivação e disponibilidade em nos auxiliar.

\section{Referências}

Barbosa, S.D.J. and Silva, B.S. da. (2010) Interação Humano Computador. Elsevier Editora Ltda., 2010.

Barbosa, S.D.J.; Paula, M.G. (2003) Designing and Evaluating Interaction as Conversation: a Modeling Language based on Semiotic Engineering. In 10th International Workshop on Design, Specification and Verification of Interactive Systems, DSV-IS 2003, Funchal, Madeira Island, Portugal, Junho 11-13, p. 16-33.

Capelao, L., Coutinho, F., Freitas, L., Pereira, K., Prates, R.O. Uma avaliação da qualidade de uso do Moodle na UFMG. In: Anais do Moodle Moot 2011, São Paulo.

de Souza, C.S. (2005) The Semiotic Engineering of Human-Computer Interaction. The MIT Press, Cambridge.

Farkas, D.K. (1998) Layering as a Safety Net for Minimalist Documentation. In Minimalism Beyond the Nurnberg Funnel. The MIT Press, Cambridge. 
Goebel, F.R. and Chichelero, R.L. (2010) Ferramenta para construçãao do conteúdo de sistemas de ajuda baseados em camadas com posterior extração de modelos resumidos de IHC. Trabalho de Conclusão II. Porto Alegre: Facin, PUCRS.

Kearsley, G. (1998) Online Help Systems: design and implementation. Ablex Publishing Corporation, Norwood, 1998.

Leite, L.L. and Silveira, M.S. "Afinando" a comunicação entre pares para melhorar a compreensão da mensagem do designer. In Anais do X Simpósio de Fatores Humanos em Sistemas Computacionais, 2011, Porto de Galinhas.

MoodleDocs: Five Key Principles. 2010. Disponível em <http://docs.moodle.org /20/en/five_key_principles>.

Moodle.org: Estatísticas Moodle. 2013.<http://moodle.org/stats>.

Salgado, T.C. and de Souza, C.S. (2004) Uma experiência de construção da ajuda online segundo o paradigma da engenharia semiótica. In Anais do VI Simpósio sobre Fatores Humanos em Sistemas Computacionais, SBC, 205-208.

Santos Jr., Mauro Francisco dos. (2009) Exploração da Comunicação Usuário-Sistema em Sistemas de Ajuda On-line. Dissertação de Mestrado. Facin, PUC-RS.

Silva, B. S. (2005) MoLIC Segunda Edição: revisão de uma linguagem para modelagem da interação humano-computador. Tese de doutorado.,Departamento de Informática. PUC-Rio.

Silva, B.S. da and Barbosa, S.D.J. (2007) Designing Human-Computer Interaction With MoLIC Diagrams: a practical guide. In C.J.P. de Lucena (ed.) Monografias em Ciência da Computação. Rio de Janeiro, RJ, 50 p.

Silva, A. P. and Farias, A. L. G. (2010) Bibliotecas para Acesso, Estruturação de Conteúdo e Navegação em Sistemas de Ajuda Online. Trabalho de Conclusão II. Facin, PUCRS.

Silveira, M. S. (2002) Metacomunicação designer-usuário na interação humanocomputador : design e construção do sistema de ajuda. Tese de doutorado. Departamento de Informática, 2002. PUC-Rio.

Silveira, M.S.; de Souza, C.S. (2002) Design de Sistemas de Ajuda Online baseado em Modelos. In: IHC2002 - V Workshop de Fatores Humanos em Sistemas Computacionais, 2002, Fortaleza.. BNB, 2002. p. 117ᄀ128.

Silveira, M.S. and Leite, L.L. (2009) Alternativas de Ajuda On-line para Ambientes de Aprendizagem Colaborativa. In Anais do XX Simpósio Brasileiro de Informática na Educação (SBIE2009), Florianópolis.

Silveira, M.S., Barbosa, S.D.J., and Souza, C.S. De. (2000) Modelo e arquitetura de sistemas de help online. In: Anais do III Workshop de Fatores Humanos em Sistemas Computacionais. Porto Alegre: SBC.

Silveira, M.S., De Souza, C. S., and Barbosa, S. D. J. (2003) Um Método da Engenharia Semiótica para a Construção de Sistemas de Ajuda Online. In: CLIHC2003 -Latin American Conference in Human-Computer Interaction, 2003, Rio de Janeiro. $167 \neg 177$. 